\title{
OPERATOR RANGES AND COMPLETELY BOUNDED HOMOMORPHISMS
}

\author{
D. BENJAMIN MATHES
}

(Communicated by John B. Conway)

\begin{abstract}
In this paper it is shown that the set of invariant operator ranges that induce completely bounded homomorphisms is a sublattice of $\mathrm{Lat}_{1 / 2} \mathfrak{A}$, when $\mathfrak{A}$ is any norm closed algebra of operators on a Hilbert space. A characterization of this sublattice is given, and several concrete examples are discussed.
\end{abstract}

\section{INTRODUCTION}

The most famous question in the theory of operator ranges is Dixmier's. If $\mathbf{H}$ is a Hilbert space, $\mathfrak{A}$ is a unital $C^{*}$-algebra of operators on $\mathbf{H}$, and $T$ is an operator on $\mathbf{H}$ whose range is invariant under every operator in $\mathfrak{A}$, Dixmier's question asks if there exists an operator $B$ in the commutant of $\mathfrak{A}$ with the same range as that of $T$. An affirmative answer is known to be true for a large class of $C^{*}$-algebras (see [6], [18]), but the general case is still unresolved. In [6] Foias proves that given an operator $T$ whose range is invariant under an algebra of operators $\mathfrak{A}$, there exists a homomorphism $\Phi_{T}$ that maps $\mathfrak{A}$ into $B(\mathbf{H})$ (the algebra of operators on $\mathbf{H}$ ), and satisfies $A T=T \Phi_{T}(A)$ for all $A \in \mathfrak{A}$. Furthermore, this homomorphism is bounded whenever $\mathfrak{A}$ is norm closed. In case $\mathfrak{A}$ is a $C^{*}$-algebra, Foias proves in the same paper that if $\Phi_{T}$ is similar to a *-homomorphism, then there exists an operator $B \in \mathfrak{A}^{\prime}$ (the commutant of $\mathfrak{A}$ ) with the same range as that of $T$. In [7] Haagerup proves that a homomorphism of a unital $C^{*}$-algebra into $B(\mathbf{H})$ is similar to a $*$-homomorphism if and only if it is completely bounded. These two results together say that Dixmier's question has an affirmative answer if and only if invariant ranges of unital $C^{*}$-algebras always induce completely bounded homomorphisms. If $\operatorname{Lat}_{1 / 2} \mathfrak{A}$ denotes the lattice of invariant operator ranges of $\mathfrak{A}$ (with intersection and linear span as lattice operations), we see from the comments above that

$$
\left\{B \mathbf{H} \mid B \in \mathfrak{A}^{\prime}\right\}=\left\{T \mathbf{H} \in \mathrm{Lat}_{1 / 2} \mathfrak{A} \mid \Phi_{T} \text { completely bounded }\right\} .
$$

Received by the editors October 6, 1988. Portions of this paper were presented on May 22, 1989 to the Canadian Operator Symposium at the University of Toronto.

1980 Mathematics Subject Classification (1985 Revision). Primary 47C05; Secondary 47A05.

Key words and phrases. Invariant operator range, completely bounded homomorphism.

The results of this paper constitute a portion of the author's $\mathrm{Ph}$. D. dissertation at the University of New Hampshire. 
Suppose now that $\mathfrak{A}$ is the algebra of analytic Toeplitz operators. In [6] Foias proves that $\{B \mathbf{H} \mid B \in \mathfrak{A}\}$ is not a lattice (it fails to be closed under linear span); thus the analog of Dixmier's question (is every element of $L^{2} t_{1 / 2} \mathfrak{A}$ the range of some operator in $\mathfrak{A}^{\prime}=\mathfrak{A}$ ) is easily answered in the negative. In [17] Roy proves that for this algebra

$$
\mathfrak{L}_{\infty} \equiv\left\{T \mathbf{H}^{(\infty)} \mid T \in B\left(\mathbf{H}^{(\infty)}, \mathbf{H}\right) ; A T=T A^{(\infty)} \text { for all } A \in \mathfrak{A}\right\}
$$

is a sublattice of $\operatorname{Lat}_{1 / 2} \mathfrak{A}$ and conjectures that it is all of $\operatorname{Lat}_{1 / 2} \mathfrak{A}$. Combining results from [11] and [14] it is immediately deduced that

$$
\mathfrak{L}_{\infty}=\left\{T \mathbf{H} \in \mathrm{Lat}_{1 / 2} \mathfrak{A} \mid \Phi_{T} \text { completely bounded }\right\} .
$$

Thus, Roy's conjecture is that every element of $\mathrm{Lat}_{1 / 2} \mathfrak{A}$ induces a completely bounded homomorphism, when $\mathfrak{A}$ is the algebra of analytic Toeplitz operators.

In this paper we prove that if $\mathfrak{A}$ is an arbitrary unital norm closed algebra of operators on a Hilbert space $\mathbf{H}$, then the subset of $\operatorname{Lat}_{1 / 2} \mathfrak{A}$ consisting of the invariant ranges that induce completely bounded homomorphisms, which we denote Lat ${ }_{c b} \mathfrak{A}$, forms a sublattice of $\operatorname{Lat}_{1 / 2} \mathfrak{A}$. We then associate a norm closed algebra $\mathfrak{B}_{\mathfrak{A}}$ with $\mathfrak{A}$ and prove that $\mathrm{Lat}_{1 / 2} \mathfrak{B}_{\mathfrak{A}}$ is always lattice isomorphic to $\mathrm{Lat}_{\mathrm{cb}} \mathfrak{A}$, under an isomorphism that restricts to an isomorphism of Lat $\mathfrak{B}_{\mathfrak{A}}$ with Lat $\mathfrak{A}$. We use this isomorphism to carry results about Lat Le $_{1 / 2} \mathfrak{A}$ over to results about $\mathrm{Lat}_{\mathrm{cb}} \mathfrak{A}$. Finally, we obtain a general representation of $\mathrm{Lat}_{\mathrm{cb}} \mathfrak{A}$ as the set of ranges of operators $T$ that satisfy $A T=T \Phi(A)$ for all $A \in \mathfrak{A}$, where $\Phi$ is a complete contraction with domain $\mathfrak{A}$.

\section{INVARIANT RANGES INDUCING COMPLETELY BOUNDED HOMOMORPHISMS}

Let $\mathfrak{A}$ be a unital norm closed algebra of operators acting on a Hilbert space H. A bounded linear map $\Phi: \mathfrak{A} \rightarrow B(\mathbf{H})$ is called completely bounded provided $\|\Phi\|_{\mathrm{cb}} \equiv \sup \left\{\left\|\Phi \otimes 1_{n}\right\| n=1,2, \ldots\right\}$ is finite, where $1_{n}$ is the identity map on $B\left(\mathbb{C}^{n}\right)$ and $\Phi \otimes 1_{n}$ acts on an elementary tensor $A \otimes M$ in $\mathfrak{A} \otimes B\left(\mathbb{C}^{n}\right)$ by $\left(\Phi \otimes 1_{n}\right)(A \otimes M)=\Phi(A) \otimes M$.

Suppose $T \in B(\mathbf{K}, \mathbf{H}), S \in B(\mathbf{M}, \mathbf{H})$ and $T \mathbf{H}=S \mathbf{H} \in \mathrm{Lat}_{1 / 2} \mathfrak{A}$. If $T_{0}$ is the restriction of $T$ to $(\operatorname{ker} T)^{\perp}$, then $\Phi_{T}(A)=T_{0}^{-1} A T$ for every $A \in \mathfrak{A}$ (see [6]). We assert that $\Phi_{T}$ is completely bounded if and only if $\Phi_{S}$ is completely bounded. Since $T_{0} \mathbf{H}=T \mathbf{H}$, we have $\Phi_{T_{0}}$ mapping $\mathfrak{A}$ into $B\left((\operatorname{ker} T)^{\perp}\right)$. The operator $T_{0}^{-1} T$ is the orthogonal projection of $\mathbf{K}$ onto $(\operatorname{ker} T)^{\perp}$, and

$$
\Phi_{T_{0}}(A)=\left.\left(T_{0}^{-1} T\right) \Phi_{T}(A)\right|_{\mathrm{ker} T^{\perp}},
$$

thus $\Phi_{T_{0}}$ is completely bounded whenever $\Phi_{T}$ is, since a compression of a completely bounded map is completely bounded. On the other hand, note that $\Phi_{T}=\Phi_{T_{0}} \oplus 0$ relative to the decomposition of $\mathbf{K}$ as $(\operatorname{ker} T)^{\perp} \oplus \operatorname{ker} T$, so $\Phi_{T}$ is completely bounded whenever $\Phi_{T_{0}}$ is. We have that $\Phi_{T_{0}}\left(\Phi_{S_{0}}\right)$ is completely bounded if and only if $\Phi_{T}\left(\Phi_{S}\right)$ is completely bounded. To prove the assertion, 
we need only prove that $\Phi_{S_{0}}$ is completely bounded if and only if $\Phi_{T_{0}}$ is; but $\Phi_{S_{0}}(A)=R^{-1} \Phi_{T_{0}}(A) R$, where $R$ is the invertible operator $R=S_{0}^{-1} T_{0}$ in $B\left((\operatorname{ker} T)^{\perp},(\operatorname{ker} S)^{\perp}\right)$. The set $\operatorname{Lat}_{1 / 2} \mathfrak{A}$ is thus partitioned into two pieces, those invariant ranges that induce completely bounded homomorphisms, and those that do not. We call the set of invariant ranges that induce completely bounded homomorphisms $\operatorname{Lat}_{\mathrm{cb}} \mathfrak{A}$ and proceed to justify the name.

Theorem 2.1. If $\mathfrak{A}$ is a norm closed unital subalgebra of $B(\mathbf{H})$, then $\operatorname{Lat}_{\mathrm{cb}} \mathfrak{A}$ is a sublattice of $\operatorname{Lat}_{1 / 2} \mathfrak{A}$.

Proof. Assume $S, T \in B(\mathbf{H})$ and $S \mathbf{H}, T \mathbf{H} \in$ Lat $_{\mathrm{cb}} \mathfrak{A}$, hence $\Phi_{S}, \Phi_{T}$, and $\boldsymbol{\Phi}_{S} \oplus \boldsymbol{\Phi}_{T}$ are completely bounded maps. Define $W: \mathbf{H}^{(2)} \rightarrow \mathbf{H}$ by $W(u, v)=S u+T v$, thus $W \mathbf{H}^{(2)}=S \mathbf{H}+T \mathbf{H}$, and

$$
\begin{aligned}
\Phi_{W}(A)(u, v) & =W_{0}^{-1} A W(u, v)=W_{0}^{-1}(A S u+A T v) \\
& =W_{0}^{-1}\left(S \Phi_{S}(A)+T \Phi_{T}(A)\right)=W_{0}^{-1} W\left(\Phi_{S} \oplus \Phi_{T}\right)(A)(u, v) .
\end{aligned}
$$

Since $W_{0}^{-1} W$ is the orthogonal projection of $\mathbf{H}^{(2)}$ onto $\operatorname{ker} W^{\perp}$, we see that $\Phi_{W}$ is a compression of the completely bounded map $\Phi_{S} \oplus \Phi_{T}$. Hence $\Phi_{W}$ is completely bounded, and $S \mathbf{H}+T \mathbf{H} \in \mathrm{Lat}_{\mathrm{cb}} \mathfrak{A}$.

We now show that $S \mathbf{H} \cap T \mathbf{H} \in \mathrm{Lat}_{\mathrm{cb}} \mathfrak{A}$. Define $Y: \mathbf{H}^{(2)} \rightarrow \mathbf{H}^{(2)}$ by the matrix

$$
Y=\left(\begin{array}{cc}
S & -T \\
0 & 0
\end{array}\right) \text {. }
$$

Let $\mathbf{K}=\operatorname{ker} Y$, define $P: \mathbf{H}^{(2)} \rightarrow \mathbf{H}$ by $P(u, v)=u$, and let $W=\left.S P\right|_{\mathbf{K}}$. Notice that $(u, v) \in \mathbf{K}$ if and only if $S u=T v$, and that $W \mathbf{K}=S \mathbf{H} \cap T \mathbf{H}$.

Suppose $(u, v) \in \mathbf{K}$. We assert that for every $A \in \mathfrak{A},\left(\Phi_{S} \oplus \Phi_{T}\right)(A)(u, v) \in \mathbf{K}$. Note that $\left(\Phi_{S} \oplus \Phi_{T}\right)(A)(u, v) \in \mathbf{K}$ if and only if $S \Phi_{S}(A) u=T \Phi_{T}(A) v$. Since $S u=T v, S \Phi_{S}(A) u=A S u=A T v=T \Phi_{T}(A) v$, and the assertion follows.

We now look at $\Phi_{W}$ :

$$
\begin{aligned}
\Phi_{W}(A)(u, v) & =W_{0}^{-1} A W(u, v)=W_{0}^{-1} A S u=W_{0}^{-1} S\left(\Phi_{S}(A) u\right) \\
& =W_{0}^{-1} W\left(\Phi_{S}(A) u, \Phi_{T}(A) v\right)=W_{0}^{-1} W\left(\Phi_{S} \oplus \Phi_{T}\right)(A)(u, v) .
\end{aligned}
$$

Once again, $\Phi_{W}$ is a compression of $\Phi_{S} \oplus \Phi_{T}$. Thus $\Phi_{W}$ is completely bounded and $S \mathbf{H} \cap T \mathbf{H} \in \mathrm{Lat}_{\mathrm{cb}} \mathfrak{A}$.

Paulsen [14] has shown that if $\mathfrak{A}$ is a $C^{*}$-algebra of operators, then the range of an operator $T$ is in Lat $_{c b} \mathfrak{A}$ if and only if the range of $T \otimes 1$ is in Lat $1 / 2$ where $\mathfrak{K}$ denotes the compact operators on an infinite dimensional separable Hilbert space $\mathbf{K}$, and 1 is the identity map on $\mathbf{K}$. In his paper, Paulsen only concerns himself with the case when $\mathfrak{A}$ is a $C^{*}$-algebra of operators, but his result is true whenever $\mathfrak{A}$ is an arbitrary norm closed algebra of operators, when the tensor product $\mathfrak{A} \otimes \mathfrak{K}$ is taken to mean the norm closed algebra generated by $\{A \otimes K \mid A \in \mathfrak{A} ; K \in \mathfrak{K}\}$ in $B(\mathbf{H} \otimes \mathbf{K})$. 
Theorem 2.2. Assume $\mathfrak{A}$ is a norm closed unital subalgebra of $B(\mathbf{H})$. Then Lat $_{\mathrm{cb}} \mathfrak{A}$ is lattice isomorphic to Lat $_{1 / 2} \mathfrak{A} \otimes \mathfrak{K}$, and the lattice isomorphism may be chosen so that the restriction to Lat $\mathfrak{A}$ is a lattice isomorphism of Lat $\mathfrak{A}$ onto Lat $\mathfrak{A} \otimes \mathfrak{K}$.

Proof. Suppose $T, S \in B(\mathbf{H})$ and $T \mathbf{H}=S \mathbf{H}$. Then it is clear that

$$
(T \otimes 1) \mathbf{H} \otimes \mathbf{K}=(S \otimes 1) \mathbf{H} \otimes \mathbf{K} .
$$

Thus if $\mathfrak{R}$ is any operator range in $\mathbf{H}$ we may define (unambiguously) $\mathfrak{R} \otimes$ 1 to be the operator range in $\mathbf{H} \otimes \mathbf{K}$ that satisfies $T \mathbf{H}=\Re$ if and only if $(T \otimes 1) \mathbf{H} \otimes \mathbf{K}=\mathfrak{R} \otimes 1$. It follows that $\mathfrak{R} \rightarrow \mathfrak{R} \otimes 1$ is a well-defined map of the lattice of all operator ranges in $\mathbf{H}$ into the lattice of operator ranges in $\mathbf{H} \otimes \mathbf{K}$. It is immediately clear that the map is injective and that if preserves range inclusion.

To prove that the lattice operations are preserved, note that $C \mathbf{H}=S \mathbf{H}+T \mathbf{H}$ is equivalent to the range inclusion statement: $S \mathbf{H}, T \mathbf{H} \subset C \mathbf{H}$ and $S \mathbf{H}$, $T H \subset B H$ implies $C H \subset B H$. This fact, together with the fact that the set $\{\mathfrak{R} \otimes 1 \mid \mathfrak{R}$ an operator range in $\mathbf{H}\}$ is a lattice (it is the set of ranges of operators from a von Neumann algebra [4], [5], [8]), implies linear span is preserved. Intersection is proved to be preserved in an analogous way. Therefore the map $\mathfrak{R} \rightarrow \mathfrak{R} \otimes 1$ is a lattice monomorphism of the lattice of all operator ranges in $\mathbf{H}$ into the lattice of all operator ranges in $\mathbf{H} \otimes \mathbf{K}$.

From Paulsen's result and what we have shown already, $R \rightarrow R \otimes 1$ is a lattice monomorphism of Lat $_{\text {cb }} \mathfrak{A}$ into Lat $_{1 / 2} \mathfrak{A} \otimes \mathfrak{K}$, and it is trivial to verify that $\mathfrak{R}$ is closed if and only if $\mathfrak{R} \otimes 1$ is closed. Thus we are finished as soon as we prove that the mapping is onto $\operatorname{Lat}_{1 / 2} \mathfrak{A} \otimes \mathfrak{K}$.

The rest of the proof uses a technique that appears in the proof of Theorem 5 of [11]. Let $\mathfrak{D} \in \mathrm{Lat}_{1 / 2} \mathfrak{A} \otimes \mathfrak{K}$, and let $\mathcal{J}$ denote the scalar operators in $B(\mathbf{H})$. Since $\mathfrak{A}$ is unital, $\mathfrak{D} \in \mathrm{Lat}_{1 / 2} \mathscr{J} \otimes \mathfrak{K}$. By a result of Ong [12], $\mathfrak{D} \in \mathrm{Lat}_{1 / 2}(\mathscr{J} \otimes \mathfrak{K})^{-W O T}$. Since $(\mathscr{J} \otimes \mathfrak{K})^{-W O T}$ is a von Neumann algebra with Schwartz's property $P$, there exists $W \in(\mathscr{J} \otimes \mathfrak{K})^{\prime}$ such that $W(\mathbf{H} \otimes \mathbf{K})=\mathfrak{D}$, by a result of Voiculescu [18]. Our result follows because

$$
(\mathscr{J} \otimes \mathfrak{K})^{\prime}=\{T \otimes 1 \mid T \in B(\mathbf{H})\} .
$$

The following corollary follows immediately from a theorem of Azoff (see [1]), who identified a sublattice $\mathfrak{L}(\mathfrak{A})$ of $\operatorname{Lat}_{1 / 2} \mathfrak{A}$ (that he calls the characteristic manifolds of $\mathfrak{A}$ ) with the property that any unital subalgebra $\mathfrak{A}$ of $\boldsymbol{B}(\mathbf{H})$ with $\mathfrak{L}(\mathfrak{A})=\{(0), \mathbf{H}\}$ must be weakly dense in $\boldsymbol{B}(\mathbf{H})$. It is easily verified that when $\mathfrak{A}$ is norm closed, one has $\mathfrak{L}(\mathfrak{A}) \subset$ Lat $_{\text {cb }} \mathfrak{A}$, from which our corollary follows. We provide the following proof to illustrate how facts about $\mathrm{Lat}_{1 / 2}$ may be carried over to $\mathrm{Lat}_{\mathrm{cb}}$.

Corollary 2.3. Assume $\mathfrak{A}$ is a weakly closed unital subalgebra of $B(\mathbf{H})$. If Lat $_{\text {cb }} \mathfrak{A}=\{(0), \mathbf{H}\}$, then $\mathfrak{A}=B(\mathbf{H})$. 
Proof. Assume $\mathfrak{A} \neq B(\mathbf{H})$. Then $(\mathfrak{A} \otimes \mathfrak{K})^{-W O T} \neq B(\mathbf{H} \otimes \mathbf{K})$, so by a theorem of Foias [6]

$$
\text { Lat }_{1 / 2}(\mathfrak{A} \otimes \mathfrak{K})^{-W O T} \neq\{(0), \mathbf{H} \otimes \mathbf{K}\} .
$$

By Theorem 2.2 it follows that Lat cb $\mathfrak{A} \neq\{(0), \mathbf{H}\}$.

Ong proved that no invariant ranges of a norm closed algebra $\mathfrak{A}$ are lost when passing to the weak closure $\mathfrak{A}^{-W O T}$, provided the unit ball of $\mathfrak{A}$ is weakly dense in the ball of $\mathfrak{A}^{- \text {WOT }}$ (see [12]). The following theorem is the analogous statement for $\operatorname{Lat}_{\mathrm{cb}} \mathfrak{A}$.

Corollary 2.4. Suppose $\mathfrak{A}$ is a norm closed unital subalgebra of $\boldsymbol{B}(\mathbf{H})$ with the property that the unit ball of $\mathfrak{A}$ is weakly dense in the unit ball of $\mathfrak{A}^{- \text {WOT }}$. Then Lat $_{\text {cb }} \mathfrak{A}=$ Lat $_{\text {cb }} \mathfrak{A}^{-W O T}$.

Proof. It is clear that $\mathrm{Lat}_{\mathrm{cb}} \mathfrak{A}^{- \text {WOT }} \subset \mathrm{Lat}_{\mathrm{cb}} \mathfrak{A}$ (since the restriction of a completely bounded map on $\mathfrak{A}^{-W O T}$ to $\mathfrak{A}$ will be completely bounded), so assume that $\mathfrak{R} \in \mathrm{Lat}_{\mathrm{cb}} \mathfrak{A}$. We have that $\mathfrak{R} \otimes 1 \in$ Lat $_{1 / 2} \mathfrak{A} \otimes \mathfrak{K}$ by Paulsen's result [14], and thus $\mathfrak{R} \otimes 1 \in \mathrm{Lat}_{1 / 2}(\mathfrak{A} \otimes \mathfrak{K})^{-W O T}$ by a result of Ong [12]. Noting that $(\mathfrak{A} \otimes \mathfrak{K})^{-W O T}=\left(\mathfrak{A}^{-W O T} \otimes \mathfrak{K}\right)^{- \text {WOT }}$ we have $\mathfrak{R} \otimes 1 \in \mathrm{Lat}_{1 / 2}\left(\mathfrak{A}^{- \text {WOT }} \otimes \mathfrak{K}\right)^{- \text {WOT }}$ and $\mathfrak{R} \in \mathrm{Lat}_{\mathrm{cb}} \mathfrak{A}^{-W O T}$, again by Paulsen's result [14].

\section{ALGEBRAS FOR WHICH EVERY INVARIANT RANGE INDUCES} A COMPLETELY BOUNDED HOMOMORPHISM

It is clear from the comments in the introduction that if a $C^{*}$-algebra $\mathfrak{A}$ yields an affirmative answer to Dixmier's operator range question, then Lat $_{1 / 2} \mathfrak{A}=$ Lat $_{c b} \mathfrak{A}$. In particular, if $\mathfrak{A}^{\prime \prime}$ has Schwartz's property $P$, then Lat $_{1 / 2} \mathfrak{A}=\operatorname{Lat}_{\mathrm{cb}} \mathfrak{A}$. It seems that every norm closed algebra $\mathfrak{A}$ that has had its invariant operator range lattice classified satisfies $\operatorname{Lat}_{1 / 2} \mathfrak{A}=\operatorname{Lat}_{\mathrm{cb}} \mathfrak{A}$. We begin with another corollary to Theorem 2.2 .

Corollary 3.1. If $\mathfrak{A}$ is a norm closed unital subalgebra of $\boldsymbol{B}(\mathbf{H})$, then

$$
\text { Lat }_{\mathrm{cb}} \mathfrak{A} \otimes \mathfrak{K}=\mathrm{Lat}_{1 / 2} \mathfrak{A} \otimes \mathfrak{K} \text {. }
$$

Proof. Assume that $\mathfrak{D} \in \mathrm{Lat}_{1 / 2} \mathfrak{A} \otimes \mathfrak{K}$. By Theorem 2.2 there exists $T \in B(\mathbf{H})$ such that $T \mathbf{H} \in \mathrm{Lat}_{\mathrm{cb}} \mathfrak{A}$ and $\mathfrak{D}=T \mathbf{H} \otimes 1=(T \otimes 1) \mathbf{H} \otimes \mathbf{K}$. However,

$$
\Phi_{T \otimes 1}(A \otimes K)=(T \otimes 1)_{0}^{-1}(A \otimes K)(T \otimes 1)=T_{0}^{-1} A T \otimes K=\Phi_{T} \otimes 1(A \otimes K),
$$

where 1 is the identity map on $B(\mathbf{K})$. Finally, $\Phi_{T} \otimes 1$ is cumpletely bounded since both $\Phi_{T}$ and 1 are (see Theorem 10.3 of [16]).

Corollary 3.2. There exists a von Neumann algebra $\mathfrak{B}$ without Schwartz's property $P$ that satisfies $\mathrm{Lat}_{\mathrm{cb}} \mathfrak{B}=\mathrm{Lat}_{1 / 2} \mathfrak{B}$.

Proof. Let $\mathfrak{A}$ be a factor in $B(\mathbf{H})$ without Schwartz's property $P$, and let $\mathfrak{B}=(\mathfrak{A} \otimes \mathfrak{K})^{-W O T}$. Then $\mathfrak{B}$ is a factor in $B(\mathbf{H} \otimes \mathbf{K})$ without Schwartz's property 
$P$. (Prove that if $\mathfrak{B}$ is an approximately finite dimensional factor, then $\mathfrak{A}$ must be an approximately finite dimensional factor; a factor has property $P$ if and only if it is approximately finite dimensional [2].) This corollary then follows from the previous corollary.

If $0 \leq T \leq 1$ and $T=\int_{[0,1]} z d E(z)$, then it is proved in [10] that every operator that leaves $T \mathbf{H}^{-}$invariant, and whose restriction to $T \mathbf{H}^{-}$is upper triangular with respect to the decomposition $\left.\bigoplus E\left(\left(\frac{1}{2}\right)^{i+1},\left(\frac{1}{2}\right)^{i}\right]\right) \mathbf{H}$, leaves $T \mathbf{H}$ invariant. Thus, if a norm closed algebra $\mathfrak{A}$ has $T \mathbf{H}^{-} \in$ Lat $\mathfrak{A}$, and its restriction to $T \mathbf{H}^{-}$is upper triangular with respect to this decomposition, then $\operatorname{ran} T \in \operatorname{Lat}_{1 / 2} \mathfrak{A}$. The following theorem asserts that invariant ranges of this kind always induce completely bounded homomorphisms.

Theorem 3.3. Assume $\mathfrak{A}$ is a norm closed unital subalgebra of $B(\mathbf{H})$, $\mathbf{M}_{i} \in$ Lat $\mathfrak{A}(i=0,1,2, \ldots), \mathbf{M}_{0} \subset \mathbf{M}_{1} \subset \mathbf{M}_{2} \subset \cdots$, and let $\mathbf{N}_{i}=\mathbf{M}_{i} \ominus \mathbf{M}_{i-1}$, $\left(\mathbf{M}_{-1}=(0) ; i=0,1,2, \ldots\right)$. Define $T \equiv \operatorname{diag}\left(1, \frac{1}{2},\left(\frac{1}{2}\right)^{2},\left(\frac{1}{2}\right)^{3}, \ldots\right)$ relative to $\oplus \mathbf{N}_{i}$ (if $x \in \mathbf{H}$, write $x=z+\sum n_{i}$ with $n_{i} \in \mathbf{N}_{i}$ and $z \in\left(\bigoplus \mathbf{N}_{i}\right)^{\perp}$; $\left.T x=\sum\left(\frac{1}{2}\right)^{i} n_{i}\right)$. Then $\operatorname{ran} T \in$ Lat $_{\mathrm{cb}} \mathfrak{A}$.

To prove this theorem, we introduce the concept of an infinite dimensional Schur product. Suppose $S \in B(\mathbf{H}), T \in B\left(\mathbf{l}^{2}\right)$, and $\lambda_{i j}=\left\langle T e_{j}, e_{i}\right\rangle$, where $\left\{\boldsymbol{e}_{i} \mid i=0,1, \ldots\right\}$ is the standard basis of $\mathbf{l}^{2}$. Assume $\mathfrak{F}=\left\{\mathbf{M}_{i} \mid i=0,1, \ldots\right\}$ is a pairwise orthogonal family of subspaces that span $\mathbf{H}$ (we call $\mathfrak{F}$ total), and $\left(S_{i j}\right)$ is the matrix of $S$ relative to this family. Then we call the matrix $\left(\lambda_{i j} S_{i j}\right)$ the Schur product of $S$ and $T$ relative to $\mathfrak{F}$ and denote it $\operatorname{Schur}_{T}(S)$. Schur products of finite matrices are discussed in [16], and the proof of the lemma below proceeds just as in the finite dimensional case.

Lemma 3.4. If $\mathfrak{F}=\left\{\mathbf{M}_{i}: i \in N\right\}$ is a total orthogonal family of subspaces of $\mathbf{H}, S \in B(\mathbf{H})$, and $T \in B\left(\mathbf{l}^{2}\right)$, then $\operatorname{Schur}_{T}(S)$ is the matrix of an operator relative to $\mathfrak{F}$ and $\operatorname{Schur}_{T}: B(\mathbf{H}) \rightarrow B(\mathbf{H})$ is a completely bounded mapping with $\left\|\operatorname{Schur}_{T}\right\|_{\mathrm{cb}} \leq\|T\|$.

Proof. Let $\Psi: B(\mathbf{H}) \rightarrow B\left(\mathbf{H} \otimes \mathbf{l}^{2}\right)$ be the mapping $\Psi(S)=S \otimes T=(1 \otimes T)(S \otimes 1)$. Then $\|\Psi\|_{\mathrm{cb}} \leq\|T\|$ ( $\Psi$ is a composition of the complete contraction $S \rightarrow$ $S \otimes 1$ with the left multiplication operator on $B\left(\mathbf{H} \otimes \mathbf{l}^{2}\right)$ defined by $W \rightarrow$ $(1 \otimes T) W$, which is completely bounded with cb-norm equal to $\|T\|)$. Assume $P_{i}$ is the orthogonal projection of $\mathbf{H}$ onto $\mathbf{M}_{i}$, so that $S_{i j}=\left.P_{i} S\right|_{\mathbf{M}_{j}}$, and define $V: \mathbf{H} \rightarrow \mathbf{H} \otimes \mathbf{l}^{2}$ by $V x=\sum P_{i} x \otimes e_{i}$. We assert that the matrix of $V^{*} \Psi(S) V$ with respect to $\mathfrak{F}$ is $\operatorname{Schur}_{T}(S)$. Suppose $m \in \mathbf{M}_{j}$; $\left(P_{i} V^{*} \Psi(S) V\right)(m)=P_{i} V^{*}(S \otimes T)\left(m \otimes e_{j}\right)=P_{i} V^{*}\left(S m \otimes T e_{j}\right)=\left(P_{i}\left\langle T e_{j}, e_{i}\right\rangle S\right)(m)$. The last equality follows from the observation that for any $x \in \mathbf{H}$,

$\left\langle P_{i} V^{*}\left(S m \otimes T e_{j}\right), x\right\rangle=\left\langle S m \otimes T e_{j}, V P_{i} x\right\rangle$

$$
=\left\langle S m, P_{i} x\right\rangle\left\langle T e_{j}, e_{i}\right\rangle=\left\langle P_{i}\left\langle T e_{j}, e_{i}\right\rangle S m, x\right\rangle .
$$


Thus

$$
\left\|\operatorname{Schur}_{T}\right\|_{\mathrm{cb}}=\left\|V^{*} \Psi V\right\|_{\mathrm{cb}} \leq\|\Psi\|_{\mathrm{cb}} \leq\|T\| .
$$

Proof of Theorem 3.3. Assume the hypothesis, so $\bigoplus \mathbf{N}_{i} \in$ Lat $\mathfrak{A}$ and the restriction of $\mathfrak{A}$ to $\bigoplus \mathbf{N}_{i}$ is upper triangular. The matrix of $T$ relative to this decomposition is diagonal, so $\Phi_{T_{0}}$ has a particularly simple form;

$$
\Phi_{T_{0}}(A)=\left(\begin{array}{cccc}
1 & 0 & 0 & \ldots \\
0 & 2 & 0 & \ldots \\
0 & 0 & 2^{2} & \ldots \\
\vdots & \vdots & \vdots & \ddots
\end{array}\right)\left(\begin{array}{cccc}
A_{00} & A_{01} & A_{02} & \cdots \\
0 & A_{11} & A_{12} & \cdots \\
0 & 0 & A_{22} & \cdots \\
\vdots & \vdots & \vdots & \ddots
\end{array}\right)\left(\begin{array}{cccc}
1 & 0 & 0 & \cdots \\
0 & \frac{1}{2} & 0 & \cdots \\
0 & 0 & \left(\frac{1}{2}\right)^{2} & \ldots \\
\vdots & \vdots & \vdots & \ddots
\end{array}\right) .
$$

When the matrices are multiplied out, we are left with

$$
\Phi_{T_{0}}(A)=\left(\begin{array}{ccccc}
A_{00} & \frac{1}{2} A_{01} & \frac{1}{2^{2}} A_{02} & \frac{1}{2^{3}} A_{03} & \cdots \\
0 & A_{11} & \frac{1}{2} A_{12} & \frac{1}{2^{2}} A_{13} & \cdots \\
0 & 0 & A_{22} & \frac{1}{2} A_{23} & \cdots \\
0 & 0 & 0 & A_{33} & \cdots \\
\vdots & \vdots & \vdots & \vdots & \ddots
\end{array}\right),
$$

which is a Schur product of $A$ (relative to $\bigoplus \mathbf{N}_{i}$ ) with the adjoint of an analytic Toeplitz operator. It follows from Lemma 3.4 that $\Phi_{T_{0}}$ is completely bounded.

Corollary 3.5. Assume $\mathfrak{A}$ is a norm closed algebra such that $\left(\mathfrak{A} \cap \mathfrak{A}^{*}\right)^{\prime \prime}$ contains a MASA $\mathfrak{M}$ and $\mathfrak{M A M} \subset \mathfrak{A}$. Then Lat $1 / 2$

Proof. By Davidson's generalization of the work of Foias and Ong (see [3], [6], [12], and [13]), all invariant ranges of $\mathfrak{A}$ are obtained as

$$
\mathfrak{R}=\left\{\sum_{i=0}^{\infty} x_{i} \mid x_{i} \in \mathbf{N}_{i}, \sum_{i=0}^{\infty}\left(2^{i}\left\|x_{j}\right\|\right)^{2}<\infty\right\}
$$

where $\mathbf{N}_{i}=\mathbf{M}_{i} \ominus \mathbf{M}_{i-1}\left(\mathbf{M}_{-1}=(0) ; i=0,1,2, \ldots\right)$, and $\left\{\mathbf{M}_{i}\right\}$ is an increasing sequence in Lat $\mathfrak{A}$. By Theorem 3.3 these are all in Lat $\mathfrak{c b}$.

\section{A REPRESENTATION OF Lat $\operatorname{cb}_{\mathfrak{A}} \mathfrak{A}$}

If $\mathfrak{A}$ is either a $C^{*}$-algebra or the algebra of analytic Toeplitz operators, then it is possible to find a Hilbert space $K$ and a complete contraction $\Phi: \mathfrak{A} \rightarrow B(\mathbf{K})$ such that

$$
\text { Lat }_{\text {cb }} \mathfrak{A}=\{T \mathbf{K} \mid T \in B(\mathbf{K}, \mathbf{H}) ; A T=T \Phi(A) \text { for all } A \in \mathfrak{A}\} \text {. }
$$

When $\mathfrak{A}$ is a $C^{*}$-algebra, then $\mathbf{K}=\mathbf{H}$ and $\Phi$ is the identity map. When $\mathfrak{A}$ is the algebra of analytic Toeplitz operators, then $K=\mathbf{H}^{(\infty)}$ and $\Phi$ is the infinite inflation map. If $\mathfrak{A}$ is an arbitrary unital norm closed subalgebra of $B(\mathbf{H}), \mathbf{K}$ is a Hilbert space, and $\Phi: \mathfrak{A} \rightarrow B(\mathbf{K})$ is a complete contraction, then define

$$
\mathfrak{L}_{\Phi} \equiv\{T \mathbf{K} \mid T \in B(\mathbf{K}, \mathbf{H}), A T=T \Phi(A) \text { for all } A \in \mathfrak{A}\}
$$

It follows immediately that $\mathfrak{L}_{\Phi} \subset \mathrm{Lat}_{\mathrm{cb}} \mathfrak{A}$. We now prove that $\Phi$ may always be chosen so that equality holds. 
Lemma 4.1. Assume $\mathfrak{A}$ is a norm closed unital subalgebra of $B(\mathbf{H}), \quad T \neq 0$, and $T \mathbf{H} \in \mathrm{Lat}_{\mathrm{cb}} \mathfrak{A}$. Then there exists a Hilbert space $\mathbf{K}$ and $S \in B(\mathbf{K}, \mathbf{H})$ such that $\operatorname{ran} S=\operatorname{ran} T$ and $\left\|\Phi_{S}\right\|_{\mathrm{cb}}=1$.

Proof. Since $\Phi_{T_{0}}: \mathfrak{A} \rightarrow B\left((\operatorname{ker} T)^{\perp}\right)$ is a completely bounded unital homomorphism, Theorem 8.1 of [16] gives us an invertible operator $R \in B\left((\operatorname{ker} T)^{\perp}\right)$ such that the map $\pi: \mathfrak{A} \rightarrow B\left((\operatorname{ker} T)^{\perp}\right)$ defined by $\pi(A)=R^{-1} \Phi_{T_{0}}(A) R$ is a complete contraction (see also [15]). Letting $S=T_{0} R$ and $\mathbf{K}=(\operatorname{ker} T)^{\perp}$, we have

$$
\pi(A)=R^{-1} \Phi_{T_{0}}(A) R=R^{-1} T_{0}^{-1} A T_{0} R=\left(T_{0} R\right)^{-1} A\left(T_{0} R\right)=\Phi_{S}(A) .
$$

It follows that $\left\|\Phi_{S}\right\|_{\mathrm{cb}} \leq 1$, and since $\Phi_{S}$ is unital, $\left\|\Phi_{S}\right\|_{\mathrm{cb}}=1$.

Theorem 4.2. Assume $\mathfrak{A}$ is a norm closed unital subalgebra of $\boldsymbol{B}(\mathbf{H})$. Then there exists a Hilbert space $\mathbf{K}$ and a complete contraction $\Phi: \mathfrak{A} \rightarrow B(\mathbf{K})$ such that $\mathrm{Lat}_{\mathrm{cb}} \mathfrak{A}=\mathfrak{L}_{\Phi}$.

Proof. For each $\mathfrak{R} \in \operatorname{Lat}_{\mathrm{cb}} \mathfrak{A}, \mathfrak{R} \neq(0)$, choose a Hilbert space $\mathbf{H}_{\mathfrak{R}}$ and $S_{\mathfrak{R}} \in B\left(\mathbf{H}_{\mathfrak{R}}, \mathbf{H}\right)$ so that $\operatorname{ran} S_{\mathfrak{R}}=\mathfrak{R}$ and $\left\|\Phi_{S_{\mathfrak{R}}}\right\|_{\mathrm{cb}}=1$ (as in Lemma 4.1); henceforth we write $\Phi_{\Re}$ in place of $\Phi_{S_{\mathfrak{R}}}$. Let $\Omega=$ Lat $_{\mathrm{cb}} \mathfrak{A} \backslash\{(0)\}$ and define

$$
\Phi \equiv \bigoplus_{\mathbf{R} \in \Omega} \Phi_{\mathfrak{R}} \quad \text { and } \quad \mathbf{K} \equiv \bigoplus_{\mathfrak{R} \in \Omega} \mathbf{H}_{\mathfrak{R}} .
$$

It follows from a "canonical shuffle" (as discussed in [16]) that $\Phi$ is completely bounded and $\|\Phi\|_{\mathrm{cb}}=1$.

Suppose $\mathfrak{R}_{0} \in \mathrm{Lat}_{\mathrm{cb}} \mathfrak{A}$; it is clear that $(0) \in \mathfrak{L}_{\Phi}$, so assume $\mathfrak{R}_{0} \neq(0)$. Define $T: \mathbf{K} \rightarrow \mathbf{H}$ by $T\left(\bigoplus x_{\mathfrak{R}}\right)=S_{\mathfrak{R}_{0}}\left(x_{\mathfrak{R}_{0}}\right) ; T$ is clearly bounded and if $A \in \mathfrak{A}$,

$$
A T\left(\bigoplus x_{\mathfrak{R}}\right)=A S_{\mathfrak{R}_{0}}\left(x_{\mathfrak{R}_{0}}\right)=S_{\mathfrak{R}_{0}} \Phi_{\mathfrak{R}_{0}}(A)\left(x_{\mathfrak{R}_{0}}\right)=T \Phi(A)\left(\bigoplus x_{\mathfrak{R}}\right) .
$$

Thus $T \mathbf{K} \in \mathfrak{L}_{\Phi}$, and clearly $T \mathbf{K}=\mathfrak{R}_{0}$.

The following corollary appears in [11] when $\mathfrak{A}$ is the algebra of analytic Toeplitz operators and $\Phi$ is the infinite inflation map. The proof is obtained by combining Theorems 2.2 and 4.2 .

Corollary 4.3. Assume $\mathfrak{A}$ is a norm closed unital subalgebra of $B(\mathbf{H})$ and $\Phi: \mathfrak{A} \rightarrow B(\mathbf{K})$ is a complete contraction such that $\mathfrak{L}_{\Phi}=\mathrm{Lat}_{\mathrm{cb}} \mathfrak{A}$. Then

$$
\text { Lat }_{1 / 2} \mathfrak{A} \otimes \mathfrak{K}=\left\{\mathfrak{R} \otimes 1 \mid \mathfrak{R} \in \mathfrak{L}_{\Phi}\right\} .
$$

\section{Questions}

The correct generalization of Dixmier's question to arbitrary unital norm closed operator algebras is now obvious: does Lat $_{1 / 2} \mathfrak{A}=\operatorname{Lat}_{\mathrm{cb}} \mathfrak{A}$ ? It seems most likely that examples where equality does not hold will be discovered.

In view of Theorem 4.2 , one might ask for a simple complete contraction with which to write $\mathfrak{L}_{\Phi}=\operatorname{Lat}_{\mathrm{cb}} \mathfrak{A}$, as we are able to do when $\mathfrak{A}$ is a $C^{*}$-algebra 
or the algebra of analytic Toeplitz operators. For example, if $\mathfrak{A}$ is the adjoint of the algebra of analytic Toeplitz operators, can a simple $\Phi$ be found such that $\mathfrak{L}_{\boldsymbol{\Phi}}=\mathrm{Lat}_{\mathrm{cb}} \mathfrak{A}$ ? The infinite inflation clearly does not work in this situation. The ranges of Hankel operators are invariant under this algebra, and the transpose map intertwines the algebra with the Hankel operators $\left(A H=H A^{T}\right.$ for all $A \in \mathfrak{A}$ and for all Hankel operators). One can prove that all of the closed invariant subspaces of $\mathfrak{A}$ are obtained as ranges of Hankel operators. Since the restriction of the transpose map to this algebra is completely isometric, the ranges of Hankel operators are all in $\operatorname{Lat}_{c b} \mathfrak{A}$. Is Lat ${ }_{c b} \mathfrak{A}$ the lattice spanned by the ranges of Hankel operators together with the ranges in $L_{\infty}$ ?

In [9] and [11], examples are given of algebras $\mathfrak{A}$ such that Lat ${ }_{c b} \mathfrak{A}$ consists of all operator ranges that may be written as $P \mathbf{M}$, where $P \in B\left(\mathbf{H}^{(\infty)}, \mathbf{H}\right)$ is the partial isometry defined by $P\left(\bigoplus x_{i}\right)=x_{0}$, and $\mathbf{M} \in$ Lat $\mathfrak{A}^{(\infty)}$. Is there a more general principle at work here?

\section{REFERENCES}

1. E. Azoff, Invariant linear manifolds and the selfadjointness of operator algebras, Amer. J. Math. 99 (1977), 121-138.

2. A. Connes, Classification of injective factors, Ann. of Math. 104 (1976), 73-115.

3. K. R. Davidson, Invariant operator ranges for reflexive algebras, J. Operator Theory 7 (1982), 101-108.

4. J. Dixmier, Étude sur les variétés et les opérateurs de Julia, Bull. Soc. Math. France 77 (1949), 11-101.

5. P. A. Fillmore and J. P. Williams, On operator ranges, Adv. in Math. 7 (1971), 254-281.

6. C. Foiaş, Invariant para-closed subspaces, Indiana Univ. Math. J. 20 (1972), 897-900.

7. U. Haagerup, Solution of the similarity problem for cyclic representations of $C^{*}$-algebras, Ann. of Math. 118 (1983), 215-240.

8. G. W. Mackey, On the domains of closed linear transformations in Hilbert space, Bull. Amer. Math. Soc. 52 (1946), 1009.

9. B. Mathes, On invariant operator ranges of abelian strictly cyclic algebras, J. Operator Theory, (to appear).

10. E Nordgren, M. Radjabalipour, H. Radjavi, and P. Rosenthal, On invariant operator ranges, Trans. Amer. Math. Soc. 251 (1979), 389-398.

11. E. Nordgren and C. Roy, On invariant ranges of the analytic Toeplitz operators, Indiana Univ. Math. J. (to appear).

12. S.-C. Ong, Operator algebras and invariant operator ranges, $\mathrm{Ph}$. D. dissertation, Dalhousie University, Halifax, Nova Scotia, 1979.

13. __ Invariant operator ranges of nest algebras, J. Operator Theory 3 (1980), 195-201.

14. V. I. Paulsen, Completely bounded maps on $C^{*}$-algebras and invariant operator ranges, Proc. Amer. Math. Soc. 86 (1982), 91-96.

15. __ Every completely polynomially bounded operator is similar to a contraction, J. Funct. Anal. 55 (1984), 1-17. 
16. __ Completely bounded maps and dilations, Pitman Research Notes in Math. Series, no. 146, Wiley, New York, 1986.

17. C. Roy, Operator ranges of shifts and $C^{*}$-algebras, Ph. D. dissertation, University of New Hampshire, Durham, N. H., 1986.

18. D. Voiculescu, Sur les sous-espaces parafermés invariants d'une algèbre de von Neumann, Bull. Sci. Math. (2) 96 (1972), 161-168.

Department of Mathematics, Dalhousie University, Halifax, Nova Scotia, Canada B3H 3J5 